\title{
Revisión sistemática de programas de prevención familiar universal: Análisis en términos de eficacia, retención y adherencia
}

\section{Systematic review of universal family prevention programs: Analysis in terms of efficacy, retention and adherence}

\author{
Jorge Nuno Negreiros de Carvalho*, Lluís Ballester Brage**, María Valero de Vicente**, \\ JoAn AMER FERnÁndeZ**. \\ * Universidade do Porto, Oporto. Portugal. \\ ** Universidad de las Islas Baleares, Palma. España.
}

\section{Resumen}

La adherencia es un aspecto importante para la eficacia de las intervenciones familiares de prevención universal de drogas. Algunas aproximaciones sugieren mejorar las evaluaciones sobre adherencia, ya que resultan parciales y no tienen en cuenta todas sus dimensiones. El objetivo del estudio es analizar las medidas de adherencia y retención utilizadas en los programas de intervención familiar para la prevención del consumo en jóvenes de 10-14 años. Para ello se revisa la literatura sobre programas universales que han obtenido buenos resultados preventivos. Las fuentes de información consultadas son: PubMed, PsycINFO (EBSCO), PsycArticles (EBSCO), Social Work abstracts (EBSCO), CINAHL (EBSCO) SocIndex (EBSCO), Scopus, Academic Search Premier (EBSCO), SCIC-ISOC, Cochrane Database of Systematic Reviews, ERIC, ScienceDirect, Web of Science, Project Cork, Researchgate y consulta expertos. Los resultados de la búsqueda muestran 21 estudios que pertenecen a 6 programas familiares: Strengthening Families Programme 10-14, Parents Who Care, Family Check-Up, Linking Lives Health, Prevention of Alcohol use in Students y Örebro Prevention Program. Los estudios analizados aportan poca información sobre los diferentes elementos involucrados en la adherencia. La retención y la atricción diferencial son los datos que aparecen con mayor frecuencia, mientras que otros aspectos como la participación activa no aparecen en los estudios. Se discuten los resultados y se realizan recomendaciones para mejorar la evaluación de la adherencia y retención en los programas de prevención familiar. Palabras clave: Adherencia; retención; programas familiares; prevención; universal.

\section{Abstract}

Adherence is an important aspect of the effectiveness of family interventions for universal drug prevention. Some approaches suggest adherence assessments should be improved because they are partial and do not take into account all dimensions. The objective of the study is to analyze adherence and retention measures used in family intervention programs for the prevention of substance use in young people aged 10-14 years. To this end, the literature was reviewed on universal programs which have obtained good preventive results. The information sources consulted are: PubMed, PsycINFO (EBSCO), PsycArticles (EBSCO), Social Work abstracts (EBSCO), CINAHL (EBSCO) SocIndex (EBSCO), Scopus, Academic Search Premier (EBSCO), SCIC-ISOC, Cochrane Database of Systematic Reviews, ERIC, ScienceDirect, Web of Science, Project Cork, Researchgate, and consultation with experts. The search results show 21 studies belonging to 6 family programs: Strengthening Families Program 10-14, Parents Who Care, Family Check-Up, Linking Lives Health, Prevention of Alcohol use in Students, and Örebro Prevention Program. The studies analyzed provide little information on the different elements involved in adherence. Retention and differential attribution are the data that appear most frequently, while other aspects such as active participation do not appear in the studies. The results are discussed and recommendations are made to improve the evaluation of adherence and retention in family prevention programs

Keywords: Adherence; retention; family-based program; prevention; universal. 


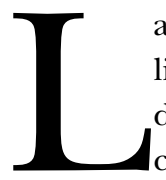

as intervenciones preventivas basadas en la familia están justificadas dada la creciente prevalencia de consumo de drogas entre la población adolescente (Rial et al., 2019; Teixidó-Compañó et al., 2019). La adherencia a los programas preventivos representa, probablemente, uno de los factores más importantes a nivel individual y familiar para asegurar niveles de eficacia adecuados (Gottfredson et al., 2015). El concepto de adherencia al tratamiento como variable que afecta a la eficacia de la intervención en prevención o en intervenciones psicosociales todavía no ha sido definido con claridad (o no al menos, tanto como se ha hecho en el ámbito de la salud) y resulta complicado diferenciarlo de otros constructos como la retención (García del Castillo, García del Castillo-López y López-Sánchez, 2014; Gearing, Townsend, Elkins, El-Bassel y Osterberg, 2014). La retención se entiende como el conjunto de medidas que informan sobre el nivel de asistencia a la intervención. En cambio, la adherencia en los tratamientos psicosociales es considerada en un sentido más amplio, como la capacidad del programa para vincular a los participantes en la intervención, e incluye la asistencia a las sesiones, la participación activa y el cumplimiento con las indicaciones del programa (Gearing et al., 2014).

Una de las causas por las que no se obtienen los resultados esperados en las intervenciones preventivas tiene que ver con la dificultad para obtener niveles altos de compromiso y asistencia por parte de las familias (Al-Halabi-Díaz y Errasti, 2009; Axford, Lehtonen, Tobin, Kaoukji y Berry, 2012; Errasti et al., 2009). La mejora de la adherencia es un tema que preocupa a los responsables técnicos y políticos, ya que supone una amenaza a la validez y la eficacia de las intervenciones (Axford et al., 2012; Byrnes, Miller, Aalborg, Plasencia y Keagy, 2010; Gearing et al., 2014; Negreiros, 2013; Spoth y Redmond, 2002). Revisiones recientes sobre programas basados en evidencias no abordan el tema de la retención y la adherencia (Lloret, Espada, Cabrera y Burkhart, 2013). Sin embargo, varios estudios sugieren las importantes implicaciones en los resultados preventivos (Al-Halabi-Diaz y Errasti, 2009; Gearing et al., 2014). Por ejemplo, se sabe que los formadores o técnicos que implementan la intervención y que proporcionan contenidos para la prevención, influyen sobre la adherencia de los participantes. En este caso, la adherencia se entiende como fidelidad al contenido, que se relaciona con la satisfacción de los participantes, con el cumplimiento de las prescripciones de la intervención y la calidad de la implementación (Byrnes et al., 2010; Orte, Ballester, Amer y Vives, 2014; Sexton y Turner, 2010). Los programas con buenos resultados en las evaluaciones de proceso presentan tasas de adherencia más altas (Haevelmann et al., 2013), lo que mejora el potencial de prevención en salud, en calidad de vida, en motivación, tanto de participantes como de profesionales, y en el uso eficiente de los recursos (Guyll, Spoth y Cornish, 2012).
La falta de adherencia en los programas preventivos provoca una reducción de los resultados esperados, así como efectos sobre el resto de los participantes, los cuales podrían perder parte de la motivación. Por lo tanto, no solo tiene efectos sobre los sujetos participantes, sino también sobre el conjunto del programa, reduciendo su credibilidad (Aarons, Hurlburt y Horwitz, 2011; Allen, Linnan y Emmons, 2012; Segrott et al., 2017). La no adherencia, además de peores resultados preventivos, genera un gasto improductivo de los recursos destinados a prevención y puede aumentar la problemática debido a la falta de atención necesaria (Gearing et al., 2014). En los últimos años, se han publicado algunas investigaciones que pretenden abordar elementos que están relacionados, como las barreras a la participación e implicación de las familias a los programas preventivos (Al-Halabi-Diaz y Errasti, 2009; Negreiros, 2013; Negreiros, Ballester, Valero, Carmo y da Gama, 2019). Por ejemplo, la adherencia en los programas preventivos universales puede verse afectada por fenómenos como la autoselección de las familias más motivadas y con mejor funcionamiento familiar previo, quedando fuera, en mayor proporción, familias que tienen mayor nivel de riesgo (Rosenman, Goates y Hill, 2012).

\section{¿Cómo medimos la adherencia al tratamiento preventivo?}

Siguiendo los resultados de revisiones recientes sobre intervenciones psicosociales, la adherencia se entiende como un constructo compuesto de varias medidas que pueden agruparse en: 1) medidas de asistencia a la sesión; 2) medidas de participación activa en la sesión; y 3) medidas de realización de tareas entre sesiones (Gearing et al., 2014). Los diferentes niveles de participación e implicación varían a lo largo de todo el proceso de intervención y para su análisis es necesario especificar momentos de evaluación e indicadores concretos (Bamberger, Coatsworth, Fosco y Ram, 2014). Actualmente, la asistencia a las sesiones (retención) es una de las medidas más frecuentes (Gearing et al., 2014), mientras que las medidas sobre los aspectos comportamentales y actitudinales de los participantes han recibido menos atención (Bamberger et al., 2014). Medir adherencia únicamente a partir de retención o asistencia resulta insuficiente, por lo que es necesario complementar con otras medidas, como la captación, el nivel de cumplimiento de la intervención, la participación activa y comprometida o la aplicación de estrategias motivacionales.

El estudio de la adherencia preventiva es importante para explicar y mejorar la capacidad del programa para producir cambios comportamentales (Gearing et al., 2014), sin embargo, falta consenso y sistematización en su estudio. Por ello, el objetivo principal de esta investigación se centra en explorar cómo se reporta y evalúa la adherencia y la retención en programas familiares de prevención universal que son considerados eficaces. El análisis de las medidas de adherencia proporciona un estándar de eva- 
luación y permite detectar aspectos ausentes y de mejora que contribuyen a la consolidación del concepto de adherencia dentro de los programas de prevención universal. Para ello se ha realizado una revisión sistemática de la literatura y se han analizado diferentes aspectos relacionados con la adherencia de los programas encontrados.

\section{Método}

\section{Criterios de inclusión y exclusión}

Para seleccionar los estudios se establecieron criterios de inclusión y exclusión, siguiendo las recomendaciones de Sánchez-Meca y Botella (2015) para la realización de revisiones sistemáticas y meta-análisis, como un elemento de garantía de calidad en la selección. Los criterios de inclusión considerados fueron publicaciones en revistas científicas entre los años 2007 y 2019, en español o inglés, sobre programas de prevención familiar universal basados en evidencias que estuvieran destinados a jóvenes de entre 10 y 14 años. Así mismo, debían informar sobre los resultados en prevención del consumo de drogas (alcohol, tabaco o drogas ilegales). Se excluyeron los estudios sin grupo control, de caso único, sin componente familiar claramente definido o falta de datos.

\section{Estrategia de búsqueda}

La presente revisión sistemática de la literatura se llevó a cabo entre febrero de 2018 y febrero de 2019. Se consultaron las siguientes bases de datos: PubMed, PsycINFO (EBSCO), PsycArticles (EBSCO), Social Work abstracts (EBSCO), CINAHL (EBSCO) SocIndex (EBSCO), Scopus, Academic Search Premier (EBSCO), SCIC-ISOC, Cochrane Database of Systematic Reviews, ERIC, ScienceDirect, Web of Science y Project Cork. La búsqueda se realizó en paralelo por dos de los autores del estudio basándose en el protocolo para la elaboración de revisiones sistemáticas de PRISMA (Urrútia y Bonfill, 2010).

En una primera fase, la búsqueda se dirigió hacia las experiencias europeas de adaptación con el Strengthening Families Program (SFP) que hubieran obtenido resultados positivos en prevención de drogas. Las palabras clave que se utilizaron fueron: Strengthening Families Program, SFP, 10 14. Se encontraron 17 artículos, de los cuales solo 7 cumplían los criterios de inclusión (véase figura 1).

En una segunda fase, debido al bajo número de estudios con resultados sobre el SFP universal, se decidió ampliar la búsqueda incluyendo el resto de los programas de prevención familiar universal consultando las mismas bases de datos. Las palabras clave fueron: prevention, universal, program,

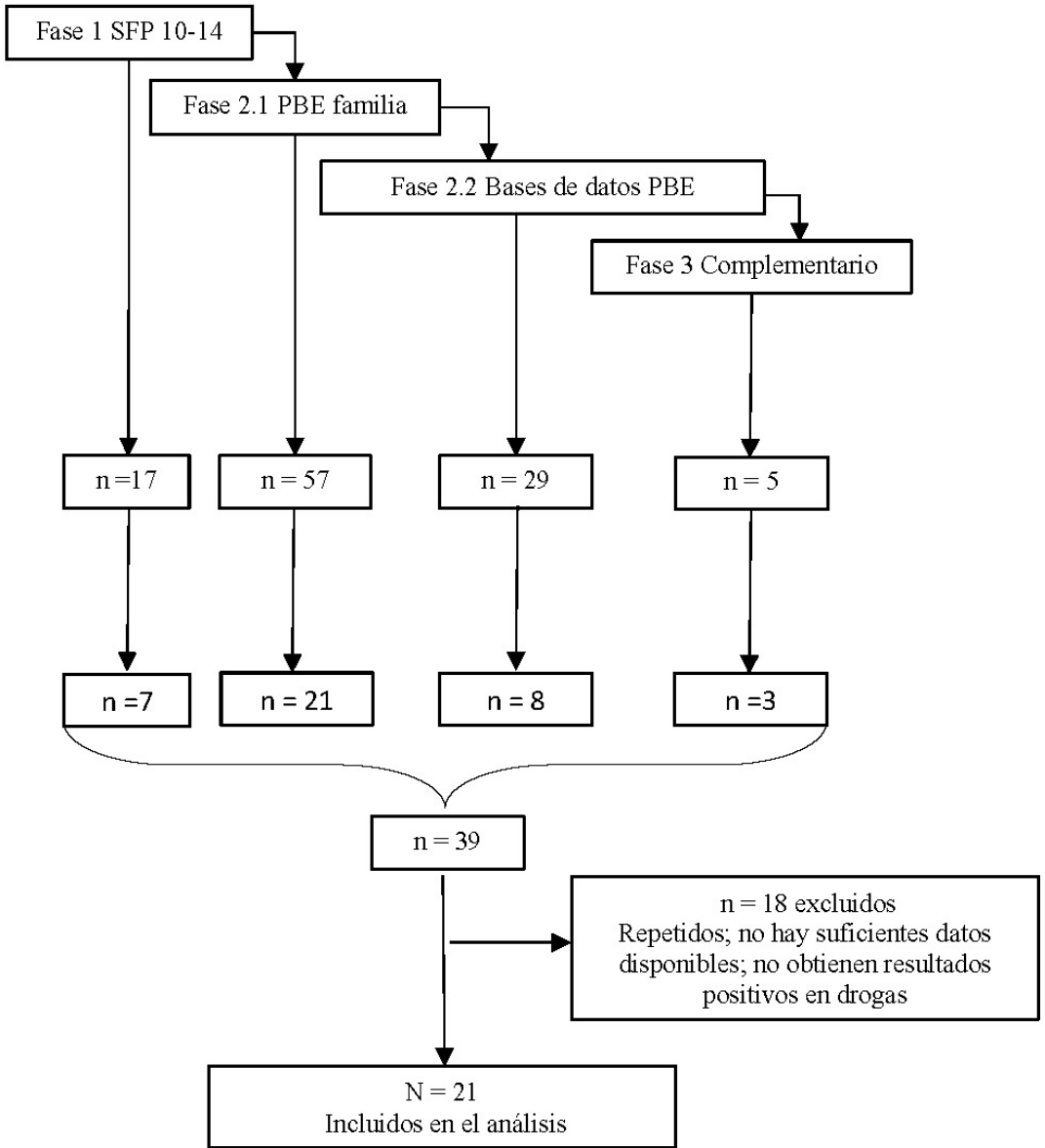

Nota. Fuente: Elaboración propia.

Figura 1. Flujograma de las fases de búsqueda y la selección de estudios. 
intervention, family-based. Como resultado de esta búsqueda, se encontraron 57 artículos y se seleccionaron 21 (véase figura 1). En esta misma fase también se consultaron los portales de referencia de Prácticas Basadas en la Evidencia (PBE): Blueprints (Universidad de Colorado-Boulder), Xchange (European Monitoring Center of Drugs and Drugs Addiction, EMCDDA) y SAMHSA (Substance Abuse and Mental Health Services Administration). En bases de datos de PBE se seleccionaron 8 artículos. La búsqueda se complementó en la tercera fase contactando directamente con diez autores y expertos de referencia en el ámbito de la prevención a través de portal académico y divulgativo ResearchGate y por correo electrónico. Se analizaron en profundidad un total de 39 artículos, de los cuáles 18 fueron excluidos por diversos motivos (véase figura 1). Finalmente, fueron incluidos en la revisión 21 estudios.

\section{Procedimiento de análisis}

Una vez seleccionados los 21 artículos, se sistematizó el análisis generando una tabla con las principales características descriptivas de los artículos (véase tabla 1). En segundo lugar, se recogieron datos sobre los efectos o resultados en la prevención (véase tabla 2). Para poder organizar los datos sobre la retención longitudinal fue necesario realizar una agrupación en función de los meses a los que se realizaba la evaluación de seguimiento (véase tabla 2 y 3). Se clasificaron las medidas de los valores de retención justo al terminar la intervención (post), los seguimientos de menos de 12 meses, los seguimientos realizados entre los 12 y los 24 meses, y los seguimientos de más de 24 meses.

\section{Resultados}

Los 21 estudios analizados corresponden a un total de 6 programas de prevención familiar universal. Los programas analizados son: Strengthening Families Programme 10-14 (SFP 10-14), Parents Who Care, Family Check-Up (FCU), Linking Lives Health, Prevention of Alcohol use in Students (PAS), y Örebro Prevention Program (ÖPP) (véase tabla 1).

Todos los estudios analizados presentaban alguna información acerca de la retención en el programa o la atrición. Sin embargo, se observa la falta de información sobre participación activa o seguimiento y aplicación de las pautas ofrecidas por la intervención. En cuanto a la calidad metodológica, destaca que del total de estudios analizados, 18 son estudios aleatorizados y 3 cuasi-experimentales. Además, todos ellos incluyen grupo control y evaluación longitudinal (véase tabla 1 ).

Teniendo en cuenta sólo los datos de los grupos de intervención se encuentran medias de retención del 86,1\% en el post, $78,9 \%$ en seguimientos inferiores a los 12 meses, de $80,81 \%$ en seguimientos de entre 12 y 24 meses, y $72 \%$ en los seguimientos superiores a los 24 meses (véase tabla 2). En la tabla 3 se pueden observar los datos en relación a la retención del grupo control. Encontramos medias de retención de $90,7 \%$ en el post, de $87,6 \%$ en el seguimiento antes de los 12 meses, $78,1 \%$ al seguimiento entre los 12 y los 24 meses, y del 70,5\% en seguimientos superiores a los 24 meses. Los mayores tiempos de seguimiento son de hasta los 21 años (Spoth, Trudeau, Shin y Redmond, 2008a; Spoth, Trudeau, Shin, Randall y Mason, 2018), y de 3 meses los seguimientos más cortos (Coombes, Allen y Foxcroft, 2012). Algunos de los estudios también reportan medidas de la retención en función de la modalidad de tratamiento, como es el caso de las comparaciones de Koning et al. (2009), de Spoth et al. (2008a), y de Spoth, Randall, Trudeau, Shin y Redmond, 2008b). Algunos estudios reportan datos por separado, para familias, padres y jóvenes, si bien esta práctica no está muy extendida, la mayoría ofrecen datos sobre cuidadores (padres-madres), jóvenes o de familias en conjunto.

Los niveles más bajos de retención coinciden con los periodos más largos en los seguimientos longitudinales (más de 24 meses), especialmente en los grupos de comparación (Koning et al., 2009; Koning, van den Eijnden, Verdurmen, Engels y Vollebergh, 2011, 2013; Spoth et al., 2008ab; Verdurmen, Koning, Vollebergh, van den Eijnden y Engels, 2014) (véase tabla 3).

En relación a la capacidad de reclutamiento del programa, solo algunos estudios reportan datos para su cálculo (Coombes et al., 2012; Haggerty, Skinner, MacKenzie y Catalano, 2007) y señalan los porcentajes de participación haciendo referencia al número de sesiones a las que han asistido. Por ejemplo, Coombes et al. en 2012 señalan que el $98 \%$ ha asistido a 5 o más sesiones, el $86 \%$ a 6 o más sesiones, y el $66 \%$ a las 7 sesiones. En esta misma línea, Haggerty et al. (2007) señalan que la media de sesiones telefónicas completadas por los padres es de 9,6 y la asistencia a la modalidad presencial es de media 4,6 sesiones. En general no se encuentra ninguna valoración acerca del grado de implicación o participación durante el desarrollo de las sesiones.

Casi todos los estudios han evaluado la atrición diferencial, encontrando diferencias entre grupos y entre condiciones de la línea base. Destaca la probabilidad diferencial de abandonar el programa durante los seguimientos si los jóvenes consumen alcohol y otras sustancias en la línea base (Baldus et al., 2016; Bodin y Strandberg, 2011; Koning et al., 2011; Koutakis, Stattin y Kerr, 2008; Spoth et al., 2008b), si los padres son indulgentes (Koutakis et al., 2008) o la percepción de riesgo, aunque estos últimos son resultados contradictorios (Bröning et al., 2017; Spoth et al., 2008a). El resto de los estudios, han examinado la atrición en relación a los porcentajes de retención en cada seguimiento (Guilamo-Ramos et al., 2010; Haggerty et al., 2007; Haggerty, Skinner, Catalano, Abbott y Crutchfield, 2015; Koning et al., 2009, 2011, 2013; Verdurmen et al., 2014). 
Tabla 1. Datos descriptivos y principales resultados de los estudios.

\begin{tabular}{|c|c|c|c|c|c|}
\hline $\begin{array}{l}\text { Nombre del } \\
\text { programa }\end{array}$ & Objetivo estudio & Muestra & Intervención & Diseño & Principales resultados \\
\hline $\begin{array}{l}\text { SFP110-14 } \\
\text { Baldus et al. } \\
2016\end{array}$ & $\begin{array}{l}\text { Evaluar los efectos de } \\
\text { la versión alemana del } \\
\text { SFP. }\end{array}$ & $\begin{array}{l}\mathrm{SFP}=147 \\
\text { Control }=145\end{array}$ & $\begin{array}{l}7 \text { sesiones semanales y } 4 \\
\text { sesiones de seguimiento. }\end{array}$ & $\begin{array}{l}\text { Ensayo aleatorizado } \\
\text { por grupos con } \\
\text { análisis longitudinal. }\end{array}$ & $\begin{array}{l}\text { Resultados positivos del SFP en } \\
\text { la prevalencia de por vida en el } \\
\text { seguimiento a } 18 \text { meses. }\end{array}$ \\
\hline $\begin{array}{l}\text { SFP 10-14 } \\
\text { Bröning et al. } \\
2017\end{array}$ & $\begin{array}{l}\text { Verificar la hipótesis } \\
\text { moderadora del riesgo. }\end{array}$ & $\begin{array}{l}\mathrm{SFP}=147 \\
\text { Control }=145\end{array}$ & $\begin{array}{l}7 \text { sesiones semanales y } 4 \\
\text { sesiones de seguimiento. }\end{array}$ & $\begin{array}{l}\text { Ensayo aleatorizado } \\
\text { por grupos con } \\
\text { análisis longitudinal. }\end{array}$ & $\begin{array}{l}\text { Pequeños efectos en niños de } \\
\text { alto riesgo a favor de SFP para la } \\
\text { abstinencia de tabaco, alcohol y } \\
\text { cannabis. }\end{array}$ \\
\hline $\begin{array}{l}\text { SFP } \mathbf{1 0 - 1 4} \\
\text { Coombes et al. } \\
2012\end{array}$ & $\begin{array}{l}\text { Examinar la versión UK } \\
\text { del SFP 10-14. }\end{array}$ & $\begin{array}{l}\text { SFP: } \\
\text { Padres: } 26 \\
\text { Jóvenes: } 34 \\
\text { Control: } \\
\text { Padres: } 27 \\
\text { Jóvenes: } 35\end{array}$ & $\begin{array}{l}7 \text { sesiones semanales y } 4 \\
\text { sesiones de seguimiento. }\end{array}$ & $\begin{array}{l}\text { Ensayo aleatorizado } \\
\text { por grupos con } \\
\text { análisis longitudinal. }\end{array}$ & - \\
\hline $\begin{array}{l}\text { SFP } 10-14 \\
\text { Foxcroft et al. } \\
2017\end{array}$ & $\begin{array}{l}\text { Evaluar la efectividad de } \\
\text { la adaptación SFP. }\end{array}$ & $\begin{array}{l}\mathrm{SFP}=223 \\
\text { Control }=229\end{array}$ & $\begin{array}{l}7 \text { sesiones semanales y } 4 \\
\text { sesiones de seguimiento, } \\
\text { sin tareas para casa. }\end{array}$ & $\begin{array}{l}\text { Ensayo aleatorizado } \\
\text { por grupos con } \\
\text { análisis longitudinal. }\end{array}$ & $\begin{array}{l}\text { No tuvieron ningún impacto } \\
\text { a los } 12 \text { o } 24 \text { meses de } \\
\text { seguimiento sobre los } \\
\text { resultados del uso indebido de } \\
\text { sustancias. }\end{array}$ \\
\hline $\begin{array}{l}\text { SFP } \mathbf{1 0 - 1 4} \\
\text { Riesch et al. } \\
2011\end{array}$ & $\begin{array}{l}\text { Examinar los efectos } \\
\text { en función de la dosis } \\
\text { recibida. }\end{array}$ & $\begin{array}{l}\mathrm{SFP}=86 \\
\text { Control=81 }\end{array}$ & $\begin{array}{l}7 \text { sesiones sin sesiones } \\
\text { de seguimiento. }\end{array}$ & $\begin{array}{l}\text { Ensayo aleatorizado } \\
\text { por grupos con } \\
\text { análisis longitudinal. }\end{array}$ & $\begin{array}{l}\text { La participación en el programa } \\
\text { completo ha sido buena. Los } \\
\text { participantes que recibieron la } \\
\text { intervención de manera parcial, } \\
\text { al contrario de lo esperado, } \\
\text { tenían niveles de consumo } \\
\text { bajos y no se observaron } \\
\text { cambios al finalizar. }\end{array}$ \\
\hline $\begin{array}{l}\text { SFP10-14 } \\
\text { Skärstrand et al. } \\
2013\end{array}$ & $\begin{array}{l}\text { Informar sobre los } \\
\text { efectos de la versión } \\
\text { sueca de la SFP 10-14. }\end{array}$ & $\begin{array}{l}\mathrm{SFP}=71 \\
\text { Control }=216\end{array}$ & $\begin{array}{l}12 \text { sesiones en dos } \\
\text { partes (la segunda parte, } \\
\text { las sesiones de refuerzo } \\
\text { son opcionales). }\end{array}$ & $\begin{array}{l}\text { Ensayo aleatorizado } \\
\text { por grupos con } \\
\text { análisis longitudinal. }\end{array}$ & $\begin{array}{l}\text { No hay diferencias significativas } \\
\text { en alcohol, tabaco, uso de } \\
\text { drogas ilícitas. }\end{array}$ \\
\hline $\begin{array}{l}\text { SFP } 10-14 \\
\text { Spoth et al. } \\
2008 a ; 2018 .\end{array}$ & $\begin{array}{l}\text { Resumir los resultados } \\
\text { de dos proyectos de } \\
\text { prevención universal. }\end{array}$ & $\begin{array}{l}\text { Estudio } 1 \\
\mathrm{SFP}=238 \\
\mathrm{PDFY}^{2}=221 \\
\text { Estudio } 2 \\
\mathrm{SFP}=137 \\
\mathrm{LST}=646\end{array}$ & $\begin{array}{l}\text { Estudio } 1 \\
\text { SFP } 7 \text { sesiones } \\
\text { semanales y } 4 \text { de } \\
\text { refuerzo. } \\
\text { PDFY } 5 \text { sesiones de } 2 \\
\text { horas centrado en los } \\
\text { factores de riesgo y } \\
\text { protección para el uso } \\
\text { de sustancias, incluida } \\
\text { la gestión familiar, la } \\
\text { vinculación entre padres } \\
\text { e hijos y comunicación. } \\
\text { Estudio } 2 \\
\text { SFP } 7 \text { sesiones } \\
\text { semanales y } 4 \text { de } \\
\text { LST } 15 \text { sesiones de } \\
40-45 \text {-minutos impartido } \\
\text { por profesores en la } \\
\text { clase, con } 5 \text { sesiones de } \\
\text { seguimiento. }\end{array}$ & $\begin{array}{l}\text { Ensayo aleatorizado } \\
\text { por grupos con } \\
\text { análisis longitudinal. }\end{array}$ & $\begin{array}{l}\text { Estudio } 1 \\
\text { Los participantes en la } \\
\text { condición de intervención } \\
\text { ISFP del } 12^{\circ} \text { grado informaron } \\
\text { significativamente menos } \\
\text { uso indebido de narcóticos, } \\
\text { y los participantes con PDFY } \\
\text { informó menos uso indebido } \\
\text { que los controles que fue } \\
\text { marginalmente significativo; } \\
\text { menos uso indebido de } \\
\text { narcóticos durante la vida y uso } \\
\text { indebido de barbitúricos que los } \\
\text { controles, pero el PDFY no fue } \\
\text { significativamente diferente. } \\
\text { Estudio } 2 \\
\text { en la evaluación del } 11 \text { o grado, } \\
\text { una cantidad significativamente } \\
\text { menor de participantes } \\
\text { de la condición LST + SFP } \\
\text { 10-14 informaron haber usado } \\
\text { medicamentos no recetados en } \\
\text { su vida útil que los controles; en } \\
\text { la evaluación de } 122^{\circ} \text { grado, la } \\
\text { diferencia entre los participantes } \\
\text { LST + SFP } 10-14 \text { y los controles } \\
\text { fue marginalmente significativa. }\end{array}$ \\
\hline $\begin{array}{l}\text { SFP } 10-14 \\
\text { Spoth et al., } \\
2008 b\end{array}$ & $\begin{array}{l}\text { Examinar la hipótesis } \\
\text { moderadora del riesgo. }\end{array}$ & $\begin{array}{l}\text { SFP+LST= } 543 \\
\text { LST }=622 \\
\text { Control }=489\end{array}$ & $\begin{array}{l}\text { SFP } 7 \text { sesiones } \\
\text { semanales y } 4 \text { de } \\
\text { LST } 15 \text { sesiones de } \\
40-45 \text {-minutos impartido } \\
\text { por profesores en la } \\
\text { clase, con } 5 \text { sesiones de } \\
\text { seguimiento. }\end{array}$ & $\begin{array}{l}\text { Ensayo aleatorizado } \\
\text { por grupos con } \\
\text { análisis longitudinal. }\end{array}$ & $\begin{array}{l}\text { Efectos significativos de la } \\
\text { intervención en } 12^{\circ} \text { grado } \\
\text { en Índice de iniciación de } \\
\text { sustancias, inicio de ebriedad, } \\
\text { inicio de cigarrillos e inicio de } \\
\text { marihuana. } \\
\text { El grupo de alto riesgo } \\
\text { mostró un menor nivel de uso } \\
\text { problemático en todas las } \\
\text { variables que el grupo de riesgo } \\
\text { de control, con una frecuencia } \\
\text { de ebriedad excepcional. }\end{array}$ \\
\hline
\end{tabular}




\begin{tabular}{|c|c|c|c|c|c|}
\hline $\begin{array}{l}\text { Parents Who } \\
\text { Care } \\
\text { Haggerty et al. } \\
2007\end{array}$ & $\begin{array}{l}\text { Evaluar el impacto de la } \\
\text { intervención. }\end{array}$ & $\begin{array}{l}\text { PWC (African- } \\
\text { American; AA) }=163 \\
\text { PWC (European- } \\
\text { American; EA) }=168 \\
\text { PWC (en grupo } \\
\text { PA) }=118 \\
\text { PWC } \\
\text { (autoadministrado } \\
\text { SA) }=107 \\
\text { Control=106 }\end{array}$ & $\begin{array}{l}\text { Formato administrado por } \\
\text { padres y adolescentes: } \\
\text { siete sesiones ( } 2 \text { a } 2,5 \mathrm{~h} \\
\text { / sesión). } \\
\text { Autoadministrado con } \\
\text { soporte telefónico (SA, } \\
\text { por sus siglas en inglés): } \\
\text { actividades del libro de } \\
\text { video y video dentro de } \\
\text { las } 10 \text { semanas, con } 62 \\
\text { actividades clave y } 4 \\
\text { horas de capacitación } \\
\text { adicional en los } \\
\text { protocolos de llamadas } \\
\text { telefónicas. }\end{array}$ & $\begin{array}{l}\text { Diseño experimental } \\
(2 \times 3 \times 4) ; \text { Línea base, } \\
\text { seguimiento a } 12 \mathrm{y} \\
24 \text { meses. }\end{array}$ & $\begin{array}{l}\text { Se detectaron efectos } \\
\text { estadísticamente significativos } \\
\text { de las intervenciones en tres } \\
\text { de los resultados examinados: } \\
\text { actitudes favorables sobre el } \\
\text { uso de sustancias, iniciación } \\
\text { del uso de sustancias o sexo y } \\
\text { comportamiento violento. }\end{array}$ \\
\hline $\begin{array}{l}\text { PWC }^{4} \\
\text { Haggerty et al. } \\
2015\end{array}$ & $\begin{array}{l}\text { Evaluar los efectos } \\
\text { a largo plazo del } \\
\text { programa Staying } \\
\text { Connected with Your } \\
\text { Teen en diferentes } \\
\text { modalidades de } \\
\text { administración. }\end{array}$ & $\begin{array}{l}\text { PWC (African- } \\
\text { American; } A A)=163 \\
\text { PWC(European- } \\
\text { American; EA) }=168 \\
\text { PWC (en grupo } \\
\text { PA) }=118 \\
\text { PWC } \\
\text { (autoadministrado } \\
\text { SA) }=107 \\
\text { Control=106 }\end{array}$ & $\begin{array}{l}\text { Formato administrado por } \\
\text { padres y adolescentes: } \\
\text { siete sesiones ( } 2 \text { a } 2,5 \mathrm{~h} \\
\text { / sesión). } \\
\text { Autoadministrado con } \\
\text { soporte telefónico (SA, } \\
\text { por sus siglas en inglés): } \\
\text { actividades del libro de } \\
\text { video y video dentro de } \\
\text { las } 10 \text { semanas, con } 62 \\
\text { actividades clave y } 4 \\
\text { horas de capacitación } \\
\text { adicional en los } \\
\text { protocolos de llamadas } \\
\text { telefónicas. }\end{array}$ & $\begin{array}{l}\text { Diseño experimental } \\
(2 \times 3 \times 4) ; 6 \text { años de } \\
\text { seguimiento. }\end{array}$ & $\begin{array}{l}\text { El efecto significativo general } \\
\text { de la intervención de la PA } \\
\text { sobre los factores estresantes } \\
\text { familiares y la frecuencia del } \\
\text { uso de drogas para los negros } \\
\text { y los blancos es un hallazgo } \\
\text { importante. Estos hallazgos } \\
\text { agregan evidencia al potencial } \\
\text { de intervenciones familiares } \\
\text { relativamente breves para influir } \\
\text { en resultados años después. } \\
\text { Hubo un efecto directo de la } \\
\text { condición de PA tanto en los } \\
\text { factores estresantes familiares } \\
\text { como en la frecuencia de } \\
\text { consumo de drogas } 6 \text { años } \\
\text { después de la intervención para } \\
\text { los jóvenes blancos y negros. } \\
\text { Aquí, examinamos sólo la } \\
\text { frecuencia de uso de drogas. }\end{array}$ \\
\hline $\begin{array}{l}\text { FCU } \\
\text { Stormshak et al. } \\
2011\end{array}$ & $\begin{array}{l}\text { Evaluar el impacto } \\
\text { y la eficacia de FCU } \\
\text { y los servicios de } \\
\text { intervención vinculados } \\
\text { para reducir las } \\
\text { conductas de riesgo } \\
\text { para la salud y promover } \\
\text { la adaptación social } \\
\text { entre los jóvenes de } \\
\text { escuela intermedia. }\end{array}$ & $\begin{array}{l}\mathrm{FCU}=385 \\
\text { Control }=207\end{array}$ & $\begin{array}{l}\text { Intervención universal } \\
\text { (información sobre } \\
\text { la crianza de los } \\
\text { hijos), intervención } \\
\text { seleccionada } \\
\text { (entrevista en tres } \\
\text { sesiones, evaluación } \\
\text { y retroalimentación } \\
\text { utilizando los principios } \\
\text { de la entrevista } \\
\text { motivacional) y apoyo } \\
\text { adicional (cada plan de } \\
\text { estudios para padres). }\end{array}$ & $\begin{array}{l}\text { Ensayo aleatorizado } \\
\text { por grupos. } \\
\text { Seguimiento de } 4 \\
\text { años. }\end{array}$ & $\begin{array}{l}\text { Reducir el crecimiento del } \\
\text { consumo de alcohol, el } \\
\text { consumo de tabaco y el } \\
\text { consumo de marihuana entre } \\
\text { los jóvenes de secundaria. Los } \\
\text { tamaños del efecto fueron los } \\
\text { siguientes: uso de cigarrillos, } \\
\text { consumo de alcohol y consumo } \\
\text { de marihuana. }\end{array}$ \\
\hline $\begin{array}{l}\text { FCU } \\
\text { Fosco et al. } \\
2013\end{array}$ & $\begin{array}{l}\text { Explorar la asociación } \\
\text { entre el control } \\
\text { con esfuerzo y } \\
\text { otros resultados de } \\
\text { comportamiento clave. }\end{array}$ & $\begin{array}{l}\mathrm{FCU}=385 \\
\text { Control }=207\end{array}$ & $\begin{array}{l}\text { Intervención universal } \\
\text { (información sobre } \\
\text { la crianza de los } \\
\text { hijos), intervención } \\
\text { seleccionada } \\
\text { (entrevista en tres } \\
\text { sesiones, evaluación } \\
\text { y retroalimentación } \\
\text { utilizando los principios } \\
\text { de la entrevista } \\
\text { motivacional) y apoyo } \\
\text { adicional (cada plan de } \\
\text { estudios para padres). }\end{array}$ & $\begin{array}{l}\text { Ensayo aleatorizado } \\
\text { por grupos. } \\
\text { Seguimiento de } 4 \\
\text { años. }\end{array}$ & $\begin{array}{l}\text { Se redujo el riesgo de } \\
\text { crecimiento y consumo de } \\
\text { alcohol, tabaco y marihuana } \\
\text { hasta el octavo grado. La } \\
\text { autorregulación se relacionó } \\
\text { con un menor crecimiento } \\
\text { en el consumo de cigarrillos, } \\
\text { consumo de alcohol y consumo } \\
\text { de marihuana en los años de } \\
\text { escuela intermedia. }\end{array}$ \\
\hline
\end{tabular}




\begin{tabular}{|c|c|c|c|c|c|}
\hline $\begin{array}{l}\text { LLH }^{6} \\
\text { Guilamo-Ramos } \\
\text { et al. } \\
2010\end{array}$ & $\begin{array}{l}\text { Evaluar la efectividad } \\
\text { de un componente } \\
\text { complementario } \\
\text { basado en padres } \\
\text { a una intervención } \\
\text { escolar para prevenir el } \\
\text { consumo de cigarrillos } \\
\text { entre los africanos. }\end{array}$ & $\begin{array}{l}\mathrm{TNT}^{7} \text { más padres } \\
=695 \\
\text { Control }=691\end{array}$ & $\begin{array}{l}2 \text { sesiones con los } \\
\text { padres, } 2 \text { sesiones de } \\
\text { refuerzo y } 2 \text { llamadas de } \\
\text { refuerzo. }\end{array}$ & $\begin{array}{l}\text { Ensayo aleatorizado } \\
\text { por grupos. } \\
\text { Seguimiento } \\
15 \text { meses } \\
\text { postintervención. }\end{array}$ & $\begin{array}{l}\text { Las probabilidades de fumar } \\
\text { cigarrillos se redujeron en un } \\
42 \% \text { para los adolescentes en } \\
\text { la condición complementaria de } \\
\text { los padres en comparación con } \\
\text { la condición de solo TNT. }\end{array}$ \\
\hline $\begin{array}{l}\text { ÖPP }^{8} \\
\text { Bodin et al. } \\
2011\end{array}$ & Evaluar la efectividad. & $\begin{array}{l}1752 \text { estudiantes } \\
\text { en } 7^{0} \text { grado }(13-16 \\
\text { años) y } 1314 \\
\text { padres. }\end{array}$ & $\begin{array}{l}\text { Reuniones de maestros } \\
\text { y padres: seis cortos ( } 20 \\
\text { minutos). }\end{array}$ & $\begin{array}{l}\text { Cuasiexperimental } \\
\text { que utiliza controles } \\
\text { emparejados con } \\
\text { un diseño pre-post, } \\
\text { intención de tratar. } \\
\text { Ensayo aleatorizado } \\
\text { grupal, con } \\
\text { escuelas asignadas } \\
\text { al azar al ÖPP o } \\
\text { sin intervención. } \\
\text { Seguimiento a } 12 \text { y } \\
30 \text { meses. }\end{array}$ & $\begin{array}{l}\text { Reducción significativa del } \\
\text { consumo de alcohol a los } 12 \\
\text { meses, pero este resultado no } \\
\text { se mantuvo a los } 30 \text { meses. }\end{array}$ \\
\hline $\begin{array}{l}\text { ÖPP } \\
\text { Koutakis et al. } \\
2008\end{array}$ & Evaluar la efectividad. & $\begin{array}{l}900 \text { estudiantes } \\
\text { (13 a } 16 \text { años) y sus } \\
\text { padres }\end{array}$ & $\begin{array}{l}\text { Los padres recibieron } \\
\text { información por correo } \\
\text { y durante las seis } \\
\text { reuniones de padres en } \\
\text { las escuelas. }\end{array}$ & $\begin{array}{l}\text { Cuasiexperimental } \\
\text { que utiliza controles } \\
\text { emparejados con } \\
\text { un diseño pre- } \\
\text { post, intención de } \\
\text { tratar. Seguimiento } \\
\text { longitudinal a 2,5 } \\
\text { años. }\end{array}$ & $\begin{array}{l}\text { Trabajar a través de los padres } \\
\text { demostró ser una forma efectiva } \\
\text { de reducir el consumo de } \\
\text { alcohol y la delincuencia en los } \\
\text { menores de edad. }\end{array}$ \\
\hline $\begin{array}{l}\text { PAS }^{9} \\
\text { Koning et al. } \\
2009\end{array}$ & $\begin{array}{l}\text { Comparar el programa } \\
\text { de prevención en } \\
\text { función de intervención } \\
\text { parental e intervención } \\
\text { estudiantil. }\end{array}$ & $\begin{array}{l}\text { Intervención con los } \\
\text { padres }=689 \\
\text { Intervención } \\
\text { estudiantes }=771 \\
\text { Combinación }=380 \\
\text { Control=779 }\end{array}$ & $\begin{array}{l}\text { Intervención parental (1 } \\
\text { sesión); o intervención } \\
\text { estudiantil ( } 4 \text { sesiones } \\
\text { digitales); o ambas } \\
\text { intervenciones } \\
\text { combinadas. }\end{array}$ & $\begin{array}{l}\text { Ensayo aleatorizado } \\
\text { por grupos. } \\
\text { Seguimiento a } 10 \text { y } \\
22 \text { meses. }\end{array}$ & $\begin{array}{l}\text { Seguimiento 1: la intervención } \\
\text { combinada de estudiantes } \\
\text { y padres mostró efectos } \\
\text { significativos en el consumo } \\
\text { de alcohol semanal en exceso, } \\
\text { el consumo semanal y la } \\
\text { frecuencia de consumo. } \\
\text { Seguimiento 2: los resultados } \\
\text { se replicaron, excepto por los } \\
\text { efectos sobre el consumo de } \\
\text { alcohol por semana. }\end{array}$ \\
\hline $\begin{array}{l}\text { PAS } \\
\text { Koning et al. } \\
2011\end{array}$ & $\begin{array}{l}\text { Evaluar los efectos de } \\
\text { la versión holandesa } \\
\text { del ÖPP. }\end{array}$ & $\begin{array}{l}13-16 \text { años } \\
\text { Muestra final }(n)= \\
2937\end{array}$ & $\begin{array}{l}\text { (1) Seis sesiones cortas } \\
\text { (20 minutos) con los } \\
\text { padres y (2) cuatro } \\
\text { lecciones digitales con } \\
\text { los estudiantes; ( } 3 \text { ) } \\
\text { intervenciones } 1 \text { y } 2 \\
\text { combinadas; y (4) el } \\
\text { currículo regular como } \\
\text { condición de control. }\end{array}$ & $\begin{array}{l}\text { Cuasiexperimental. } \\
\text { Seguimiento a } 34 \\
\text { meses. }\end{array}$ & $\begin{array}{l}\text { A los } 34 \text { meses de seguimiento, } \\
\text { los efectos significativos de la } \\
\text { intervención PAS combinada } \\
\text { (padres y alumnos). El } \\
\text { inicio del consumo excesivo } \\
\text { de bebidas semanales } \\
\text { y semanales se redujo } \\
\text { significativamente en un 12,4\% } \\
\text { y un } 10,5 \% \text {, respectivamente, } \\
\text { en adolescentes. No se } \\
\text { encontraron efectos de las } \\
\text { intervenciones separadas. }\end{array}$ \\
\hline $\begin{array}{l}\text { PAS } \\
\text { Koning et al. } \\
2013\end{array}$ & $\begin{array}{l}\text { Analizar la efectividad } \\
\text { del PAS. }\end{array}$ & $\begin{array}{l}\text { Intervención } \\
\text { parental= } 254 \\
\text { Intervención } \\
\text { estudiantes }=291 \\
\text { Intervención } \\
\text { combinada= } 193 \\
\text { Control= } 326\end{array}$ & $\begin{array}{l}\text { Intervención parental (1 } \\
\text { sesión); o intervención } \\
\text { estudiantil ( } 4 \text { sesiones } \\
\text { digitales); o ambas } \\
\text { intervenciones } \\
\text { combinadas. }\end{array}$ & $\begin{array}{l}\text { Ensayo aleatorizado } \\
\text { por grupos. } \\
\text { Seguimiento a } 50 \\
\text { meses. }\end{array}$ & $\begin{array}{l}\text { La intervención combinada } \\
\text { reducía la prevalencia de } \\
\text { consumo excesivo de alcohol } \\
\text { durante el fin de semana y la } \\
\text { cantidad de consumo de alcohol } \\
\text { en general. }\end{array}$ \\
\hline $\begin{array}{l}\text { PAS } \\
\text { Verdurmen et al. } \\
2004\end{array}$ & $\begin{array}{l}\text { Examinar el impacto y } \\
\text { los efectos diferenciales } \\
\text { de los moderadores. }\end{array}$ & $\begin{array}{l}\text { Intervención } \\
\text { parental= } 608 \\
\text { Intervención } \\
\text { estudiantes }=675 \\
\text { Intervención } \\
\text { combinada }=812 \\
\text { Control=935 }\end{array}$ & $\begin{array}{l}\text { Intervención parental (1 } \\
\text { sesión); o intervención } \\
\text { estudiantil ( } 4 \text { sesiones } \\
\text { digitales); o ambas } \\
\text { intervenciones } \\
\text { combinadas. }\end{array}$ & $\begin{array}{l}\text { Ensayo aleatorizado } \\
\text { por grupos. } \\
\text { Seguimiento a } 22 \\
\text { meses. }\end{array}$ & $\begin{array}{l}\text { La intervención combinada } \\
\text { retrasó el inicio del consumo } \\
\text { semanal de alcohol en } \\
\text { la población general } \\
\text { de adolescentes, y fue } \\
\text { particularmente eficaz para } \\
\text { retrasar el inicio del consumo } \\
\text { excesivo de alcohol semanal } \\
\text { en una submuestra de mayor } \\
\text { riesgo. }\end{array}$ \\
\hline
\end{tabular}


Tabla 2. Medidas sobre retención en los grupos de intervención y porcentajes de retención.

\begin{tabular}{|c|c|c|c|c|c|}
\hline \multirow{2}{*}{ Programa } & \multirow{2}{*}{ Referencias } & \multirow{2}{*}{ Post } & \multicolumn{3}{|c|}{ Seguimientos } \\
\hline & & & Inferior a 12 meses & De 12 a 24 meses & Más de 24 meses \\
\hline \multirow[t]{7}{*}{ SFP 10-14 } & $\begin{array}{l}\text { Baldus et al. 2016; } \\
\text { Bröning et al. } 2017\end{array}$ & Familias: 139 (94,5\%) & $\begin{array}{l}6 \text { meses } \\
\text { Familias: } 136(92,5 \%)\end{array}$ & $\begin{array}{l}18 \text { meses } \\
\text { Familias: } 135(91,8 \%)\end{array}$ & \\
\hline & $\begin{array}{l}\text { Coombes et al. } \\
2012\end{array}$ & $\begin{array}{l}\text { Padres: } 23 \text { (88,4\%) } \\
\text { Jóvenes: } 24(70,5 \%)\end{array}$ & $\begin{array}{l}3 \text { meses } \\
\text { Padres: } 23(88,4 \%) \\
\text { Jóvenes: } 21(61,7 \%)\end{array}$ & & \\
\hline & $\begin{array}{l}\text { Foxcroft et al. } \\
2017\end{array}$ & & & $\begin{array}{l}12 \text { meses } \\
\text { Familias: } 203(69 \%) \\
24 \text { meses } \\
\text { Familias: } 160(52 \%)\end{array}$ & \\
\hline & $\begin{array}{l}\text { Riesch et al. } \\
2011\end{array}$ & Familias: 66 (76,7\%) & $\begin{array}{l}6 \text { meses } \\
\text { Familias: } 66(76,7 \%)\end{array}$ & & \\
\hline & $\begin{array}{l}\text { Skärstrand et al. } \\
2013\end{array}$ & & & $\begin{array}{l}12 \text { meses } \\
\text { Jóvenes: } 320(95,5 \%) \\
24 \text { meses } \\
\text { Jóvenes: } 288(87,8 \%)\end{array}$ & $\begin{array}{l}48 \text { meses } \\
\text { Jóvenes: } 283 \text { (86,2\%) }\end{array}$ \\
\hline & $\begin{array}{l}\text { Spoth et al. 2008a; } \\
\text { Spoth et al. } 2018 \\
\text { Estudio } 1\end{array}$ & $\begin{array}{l}\text { SFP: } 188 \text { (78,9\%) } \\
\text { PDFY:177 (80\%) }\end{array}$ & & $\begin{array}{l}12 \text { meses } \\
\text { SFP:161 (67,6\%) } \\
\text { PDFY: } 155(70 \%) \\
24 \text { meses } \\
\text { SFP: } 152(63,8 \%) \\
\text { PDFY:145 }(65,6 \%)\end{array}$ & $\begin{array}{l}32 \text { meses } \\
\text { SFP:152 }(63,8 \%) \\
\text { PDFY:144 (65,1\%) } \\
72 \text { meses } \\
\text { SFP:151 }(63,8 \%) \\
48 \text { meses } \\
\text { SFP: } 151(63,4 \%) \\
\text { PDFY:149 }(67,4 \%) \\
\text { A los } 21 \text { años } \\
\text { SFP: } 170(71,4 \%) \\
\text { PDFY:152 }(68,7 \%)\end{array}$ \\
\hline & $\begin{array}{l}\text { Spoth et al. 2008a; } \\
\text { Spoth et al. 2008b } \\
\text { Estudio } 2\end{array}$ & $\begin{array}{l}\text { SFP+LST: } 546 \text { jóvenes } \\
(94,7 \%) \\
\text { LST:615 jóvenes } \\
(95,2 \%)\end{array}$ & & $\begin{array}{l}12 \text { meses } \\
\text { SFP+LST: } 557 \text { jóvenes } \\
\text { (96,7\%) } \\
\text { LST:554 jóvenes }(85,7 \%) \\
24 \text { meses } \\
\text { SFP+LST: } 552(95,8 \%) \\
\text { LST:532 }(82,3 \%)\end{array}$ & $\begin{array}{l}32 \text { meses } \\
\text { SFP+LST:516 (89,5\%) } \\
\text { LST: } 474(67,1 \%) \\
\mathbf{4 8} \text { meses } \\
\text { SFP+LST: } 444(77,1 \%) \\
\text { LST: } 425(65,7 \%)\end{array}$ \\
\hline PWC & $\begin{array}{l}\text { Haggerty et al. } 2007 \text {, } \\
2015 .\end{array}$ & $\begin{array}{l}\text { SA:102 (96,2\%) } \\
\text { PA:107 (90,6\%) }\end{array}$ & & $\begin{array}{l}12 \text { meses } \\
\text { SA: } 100 \text { familias }(94,3 \%) \\
\text { PA: } 107 \text { familias }(90,6 \%) \\
24 \text { meses } \\
\text { SA: } 93 \text { familias }(87,7 \%) \\
\text { PA: } 109 \text { familias }(92,3 \%)\end{array}$ & \\
\hline FCU & $\begin{array}{l}\text { Van Ryzin et al. } 2012 \text {; } \\
\text { Stormshak et al. } \\
\text { 2011; Fosco et al. } \\
\text { 2013. }\end{array}$ & & 287 familias $(74,3 \%)$ & & \\
\hline LLV & $\begin{array}{l}\text { Guilamo-Ramos et al. } \\
2010\end{array}$ & & & $\begin{array}{l}15 \text { meses } \\
554 \text { familias }(79,2 \%)\end{array}$ & \\
\hline ÖPP & Bodin et al. 2011 & & & $\begin{array}{l}12 \text { meses } \\
835 \text { jóvenes }(93,5 \%)\end{array}$ & $\begin{array}{l}\mathbf{3 0} \text { meses } \\
798 \text { jóvenes }(87,1 \%)\end{array}$ \\
\hline & Koutakis et al. 2008 & & & $\begin{array}{l}\mathbf{1 2} \text { meses } \\
317 \text { jóvenes }(80,6 \%) \\
256 \text { padres }(75,5 \%) \\
\mathbf{2 4} \text { meses } \\
339 \text { jóvenes }(86,2 \%) \\
264 \text { padres }(77,8 \%)\end{array}$ & \\
\hline PAS & $\begin{array}{l}\text { Koning et al. 2009, } \\
\text { 2011, 2013; } \\
\text { Verdurmen et al. } 2014\end{array}$ & $\begin{array}{l}689 \text { padres }(86 \%) \\
771 \text { jóvenes }(81,8 \%) \\
698 \text { combinado } \\
(85,9 \%)\end{array}$ & $\begin{array}{l}10 \text { meses } \\
655 \text { padres }(81,7 \%) \\
730 \text { jóvenes }(77,4 \%) \\
639 \text { combinado }(78,6 \%)\end{array}$ & $\begin{array}{l}22 \text { meses } \\
608 \text { padres }(75,9 \%) \\
675 \text { jóvenes }(71,6 \%) \\
588 \text { combinado }(72,4 \%)\end{array}$ & \\
\hline & & $M=86,1$ & $M=78,9$ & $M=80,81$ & $M=72$ \\
\hline
\end{tabular}

Nota. Fuente: elaboración propia. 
Tabla 3. Medidas sobre retención en los grupos de comparación y porcentajes de retención.

\begin{tabular}{|c|c|c|c|c|c|}
\hline \multirow{2}{*}{ Programa } & \multirow{2}{*}{ Referencias } & \multirow{2}{*}{ Post } & \multicolumn{3}{|c|}{ Seguimientos } \\
\hline & & & Inferior a 12 meses & De 12 a 24 meses & Más de 24 meses \\
\hline \multirow[t]{7}{*}{ SFP 10-14 } & $\begin{array}{l}\text { Baldus et al. } 2016 \\
\text { Bröning et al. } 2017\end{array}$ & Familias: 137 (94,4\%) & $\begin{array}{l}6 \text { meses } \\
\text { Familias: } 132 \text { (91\%) }\end{array}$ & $\begin{array}{l}18 \text { meses } \\
\text { Familias: } 127(87,5 \%)\end{array}$ & \\
\hline & $\begin{array}{l}\text { Coombes et al. } \\
2012\end{array}$ & $\begin{array}{l}\text { Padres: } 27 \text { (100\%) } \\
\text { Jóvenes: } 35 \text { (100\%) }\end{array}$ & $\begin{array}{l}3 \text { meses } \\
\text { Padres: } 27(100 \%) \\
\text { Jóvenes: } 35(100 \%)\end{array}$ & & \\
\hline & $\begin{array}{l}\text { Foxcroft et al. } \\
2017\end{array}$ & & & $\begin{array}{l}12 \text { meses } \\
\text { Familias: } 178(78 \%) \\
24 \text { meses } \\
\text { Familias: } 146(65 \%)\end{array}$ & \\
\hline & Riesch et al. 2011 & Familias: 66 (81,4\%) & $\begin{array}{l}6 \text { meses } \\
\text { Familias: } 59(72,8 \%)\end{array}$ & & \\
\hline & $\begin{array}{l}\text { Skärstrand et al. } \\
2013\end{array}$ & & & $\begin{array}{l}12 \text { meses } \\
\text { Jóvenes: } 188(97,4 \%) \\
24 \text { meses } \\
\text { Jóvenes: } 177(91,7 \%)\end{array}$ & $\begin{array}{l}48 \text { meses } \\
\text { Jóvenes: } 164 \text { (84,9\%) }\end{array}$ \\
\hline & $\begin{array}{l}\text { Spoth et al. 2008a } \\
\text { Spoth et al. } 2019 \\
\text { Estudio } 1\end{array}$ & Familias: 186 (89,4\%) & & $\begin{array}{l}12 \text { meses } \\
\text { Familias: } 156(75 \%) \\
24 \text { meses } \\
\text { Familias: } 141(67,7 \%) \\
32 \text { meses } \\
\text { Familias: } 151(72,5 \%)\end{array}$ & $\begin{array}{l}48 \text { meses } \\
\text { Familias: } 157(75,4 \%) \\
72 \text { meses } \\
\text { Familias:157 (75,4\%) } \\
\text { A los } 21 \text { años } \\
\text { Familias: } 161(77,4 \%)\end{array}$ \\
\hline & $\begin{array}{l}\text { Spoth et al. 2008ab } \\
\text { Estudio } 2\end{array}$ & Jóvenes: 491 (78,5\%) & & $\begin{array}{l}12 \text { meses } \\
\text { Jóvenes: } 479(76,6 \%) \\
24 \text { meses } \\
\text { Jóvenes: } 460(73,6 \%)\end{array}$ & $\begin{array}{l}32 \text { meses } \\
\text { Jóvenes: } 452(72,3 \%) \\
48 \text { meses } \\
\text { Jóvenes: } 343(54,8 \%)\end{array}$ \\
\hline PWC & $\begin{array}{l}\text { Haggerty et al. } \\
2007,2015 .\end{array}$ & Familias: 105 (99\%) & & $\begin{array}{l}\mathbf{1 2} \text { meses } \\
99 \text { familias }(93,3 \%) \\
\mathbf{2 4} \text { meses } \\
101 \text { familias }(95,2 \%)\end{array}$ & \\
\hline FCU & $\begin{array}{l}\text { Van Ryzin et al. 2012; } \\
\text { Stormshak et al. } \\
\text { 2011; Fosco et al. } \\
2013 \text {. }\end{array}$ & & 172 familias (83\%) & & \\
\hline LLV & $\begin{array}{l}\text { Guilamo-Ramos et al. } \\
2010\end{array}$ & & & $\begin{array}{l}15 \text { meses } \\
542 \text { familias }(78,4 \%)\end{array}$ & \\
\hline \multirow[t]{2}{*}{ ÖPP } & $\begin{array}{l}\text { Bodin et al. } \\
2011\end{array}$ & & & $\begin{array}{l}12 \text { meses } \\
778 \text { jóvenes }(92,8 \%)\end{array}$ & $\begin{array}{l}30 \text { meses } \\
750 \text { jóvenes }(87,3 \%)\end{array}$ \\
\hline & $\begin{array}{l}\text { Koutakis et al. } \\
2008\end{array}$ & & & $\begin{array}{l}\mathbf{1 2} \text { meses } \\
336 \text { jóvenes }(80,3 \%) \\
268 \text { padres }(85,8 \%) \\
\mathbf{2 4} \text { meses } \\
366 \text { jóvenes }(87,5 \%)\end{array}$ & \\
\hline \multirow[t]{2}{*}{ PAS } & $\begin{array}{l}\text { Koning et al. 2009, } \\
2011,2013 ; \\
\text { Verdurmen et al. } \\
2014\end{array}$ & 779 controles $(83,3 \%)$ & $\begin{array}{l}10 \text { meses } \\
747 \text { controles }(79,8 \%)\end{array}$ & $\begin{array}{l}22 \text { meses } \\
699 \text { controles }(74,7 \%)\end{array}$ & $\begin{array}{l}\mathbf{3 4} \text { meses } \\
677 \text { controles }(72,4 \%) \\
\mathbf{5 0} \text { meses } \\
326 \text { controles }(34,8 \%)\end{array}$ \\
\hline & & $M=90,7$ & $M=87,6$ & $M=78,1$ & $M=70,5$ \\
\hline
\end{tabular}

Nota. Fuente: elaboración propia. 
En cuanto a los factores que se relacionan con la mejora de la adherencia y la retención (véase tabla 4), encontramos que cuatro (Coombes et al., 2012; Haggerty et al., 2007, 2015; Riesch et al., 2011) de los 21 estudios (19\%) señalan proporcionar ayudas y medios para favorecer la asistencia al programa (servicio de guardería, billetes de transporte, horario pactado, etc.). Respecto al uso de incentivos, 7 (Baldus et al., 2016; Coombes et al., 2012; Haggerty et al., 2007, 2015; Riesch et al., 2011; Stormshak et al., 2011; Van Ryzin, Stormshak y Dishion, 2012) de los 21 estudios $(33,3 \%)$ reportan la utilización de incentivos, ya sean económicos, prestaciones o recursos educativos varios. Otro de los aspectos a resaltar, son los seguimientos individualizados, recordatorios y llamadas telefónicas para incentivar la participación. De los 21 estudios, 8 (38\%) señala que han realizado estas tareas durante el programa. En relación a la formación de formadores, todos los programas refieren haber realizado formación específica, a excepción de dos estudios que no hacen ninguna referencia a la formación de los profesionales (Koning et al., 2011; Van Ryzin et al., 2012). La formación va desde tres horas y media de duración (Verdurmen et al., 2014), pasando por varios días (Coombes et al., 2012; Koutakis et al., 2008), hasta una semana (Fosco, Frank, Stormshak y Dishion, 2013; Stormshak et al., 2011). Sin embargo, ninguno de los estudios, a excepción del de Coombes et al. (2012), hace referencia a las características que debe de tener el formador para conectar y favorecer la adherencia de los participantes. Además, se encontró que 15 estudios trabajan a partir de contenido estructurado y manualizado $(71,4 \%)$, algunos de estos materiales están adaptados (Bröning et al., 2017; Foxcroft, Callen, Davies y Okulicz-Kozaryn, 2017; Koning et al., 2009; Skärstrand, Sundell y Andréasson, 2014). En cuanto al lugar de implementación, todos los estudios se llevan a cabo en el contexto escolar y sólo algunas de las modalidades de comparación se llevan a cabo en otros contextos (Haggerty et al., 2007, 2015; Riesch et al., 2011).

Tabla 4. Factores relacionados con la mejora de la adherencia y la retención.

\begin{tabular}{|c|c|c|c|c|c|c|c|}
\hline Programa & Referencia & $\begin{array}{c}\text { Ayudas } \\
\text { asistencia }\end{array}$ & $\begin{array}{c}\text { Incentivos } \\
\text { económicos } \\
\text { u otros }\end{array}$ & Meriendas & $\begin{array}{l}\text { Recordatorios } \\
\text { telefónicos }\end{array}$ & Formación de formadores & Manual \\
\hline \multirow[t]{8}{*}{ SFP 10-14 } & Baldus et al. 2016 & & $x$ & $x$ & & $x$ & $x$ \\
\hline & Bröning et al. 2017 & & & $x$ & & $x$ & $\mathrm{x}$ adaptado \\
\hline & Coombes et al. 2012 & $x$ & $x$ & $x$ & $x$ & 3 días & $x$ \\
\hline & Foxcroft et al. 2017 & & & & & $x$ & $\mathrm{x}$ adaptado \\
\hline & Riesch et al. 2011 & $x$ & $x$ & $x$ & $x$ & $x$ & \\
\hline & Skärstrand et al. 2013 & & & & & x (profesor y apoyo) & $\mathrm{x}$ adaptado \\
\hline & $\begin{array}{l}\text { Spoth et al. 2008a } \\
\text { Spoth et al. } 2019\end{array}$ & & & & & $x$ & \\
\hline & Spoth et al. $2008 \mathrm{~b}$ & & & & & 3 días & $x$ \\
\hline PWC & Haggerty et al. 2007 & $x$ & $x$ & & $\mathrm{x}$ & $20 \mathrm{~h}$ & $x$ \\
\hline \multirow[t]{3}{*}{ FCU } & Van Ryzin et al. 2012 & & $x$ & $x$ & & & $x$ \\
\hline & Stormshak et al. 2011 & & $x$ & $x$ & & 1 semana y seguimientos & $x$ \\
\hline & Fosco et al. 2013 & & & & & 1 semana y seguimientos & $x$ \\
\hline LLV & Guilamo-Ramos et al. 2010 & & & & $\mathrm{x}$ & & $\mathrm{x}$ \\
\hline \multirow[t]{2}{*}{ ÖPP } & Bodin et al. 2011 & & & & & $x$ & \\
\hline & Koutakis et al. 2008 & & & & & 2 días & \\
\hline \multirow[t]{4}{*}{ PAS } & Koning et al. 2009 & & & & $x$ & $x$ & $\begin{array}{c}\text { x adaptado del } \\
\text { ÖPP }\end{array}$ \\
\hline & Koning et al. 2011, 2013 & & & & $x$ & $x$ & \\
\hline & Verdurmen et al. 2014 & & & & $x$ & $x(4$ sesiones de $50 \mathrm{~min}$ ) & \\
\hline & Koning et al. 2011 & & & & & & \\
\hline
\end{tabular}

Nota. Fuente: elaboración propia. 


\section{Discusión y conclusiones}

Los programas de prevención familiar universal eficaces analizados muestran buena capacidad de retención, tanto a corto como a largo plazo, y además, cumplen con el máximo estándar de calidad metodológica (estudios aleatorizados, grupo control y seguimientos longitudinales). Sin embargo, el análisis de la eficacia preventiva necesita del estudio de diferentes niveles de adherencia a nivel de asistencia a las sesiones, de implicación dentro de las sesiones y entre sesiones (Gearing et al., 2014). A nivel general se observa que no hay un procedimiento estándar para informar sobre estos aspectos (Bamberger et al., 2014; Gearing et al., 2014).

En cuanto a los datos sobre adherencia a partir de medidas de asistencia, los programas analizados incluyen, en su mayoría, medidas sobre la asistencia a sesiones, reportando porcentajes altos de retención en los seguimientos longitudinales, especialmente en los grupos experimentales. En cambio, estos datos no se desagregan por tipo de participante (cuidador, jóvenes, o familias) o por modalidad de tratamiento (presencial, online, etc.), lo que tampoco permite analizar la capacidad de reclutamiento de los programas (pocos de ellos facilitan información sobre número de invitaciones o muestra disponible para la captación).

El uso de medidas de atricción diferencial está más extendido y aporta información relevante para el estudio de la adherencia, especialmente en los grupos experimentales. Estos resultados pueden ser útiles para mejorar la comprensión de elementos como la autoselección (Bröning et al., 2017; Rosenman et al., 2012; Spoth et al., 2008a) o como el consumo de drogas previo (Baldus et al., 2016; Bodin et al., 2011; Koning et al., 2011; Koutakis et al., 2008; Spoth et al., 2008b), para predecir el abandono prematuro del programa, especialmente en prevención universal.

El registro de asistencia la medida más frecuente, porque es fácil de recoger y normalmente se hace al final de la intervención, por lo que no es muy útil para introducir mejoras o cambios en el desarrollo del programa (Gearing et al., 2014). Así mismo, podría observarse el mismo nivel de asistencia a sesiones, pero con patrones diferentes de adherencia que reflejen resultados diferentes (Ballester, Valero, Orte y Amer, 2018).

Otras medidas, como la calidad de la participación o la realización de las tareas prescritas, son más difíciles de evaluar y por tanto se observan en menor medida (Bamberger et al., 2014; Gearing et al., 2014). En el caso de la calidad de la participación se podría evaluar mediante el registro por parte de observadores externos o del propio formador, así como también la valoración del propio usuario, de la calidad de las aportaciones realizadas a la sesión o de la vinculación emocional entre profesional y participantes. Para la evaluación del seguimiento de las prescripciones conductuales, encargadas de consolidar y generalizar los aprendizajes al contexto natural o la vida cotidiana, pueden estable- cerse controles sobre las tareas realizadas en modo de fichas, diarios u otros productos tangibles (Gearing et al., 2014).

La no adherencia afecta a la eficacia de las intervenciones y reduce el potencial impacto de los programas de prevención familiar universal (Bamberger et al., 2014; García-del-Castillo et al., 2014). Especialmente cuando la demanda de participación en el programa es alta (Negreiros, 2013) o cuando la percepción de riesgo es baja, como es el caso de la prevención universal (Bröning et al., 2017; Rosenman et al., 2012).

Entre las principales limitaciones del estudio, destaca la imposibilidad de realizar un meta-análisis debido al bajo tamaño muestral de estudios y la falta de información para poder realizar los cálculos sobre los tamaños del efecto. Otra de las limitaciones importantes tiene que ver con el contenido específico, ya que sólo se han analizado programas familiares de prevención universal que mostraran resultados positivos en la reducción del consumo de drogas.

En cuanto a las recomendaciones futuras, cabe señalar que se observan diferencias a la hora de proporcionar información sobre la inclusión de elementos o factores que la bibliografía reconoce que mejoran o incrementan la adherencia y la retención como: la fidelidad al contenido, la utilización de manuales estandarizados, los incentivos o las meriendas, la formación de formadores, la eliminación de barreras logísticas, o los recordatorios y seguimientos de la participación (Al-Halabi-Diaz y Errasti, 2009; Byrnes et al., 2010; Haevelmann et al., 2013; Kumpfer, 2008; Negreiros et al., 2019; Sexton y Turner, 2010; Orte et al., 2014). Por ello, es recomendable que aquellos programas que incluyan alguna de estas medidas reporten los datos necesarios sobre número de seguimientos o recordatorios telefónicos, número de ayudas a la asistencia, incentivos, formación de formadores, etc., para poder evaluar su impacto en los resultados de adherencia y participación (Schwalbe y Gearing, 2012).

Además, la bibliografía señala que la adherencia al programa aumenta si es estimulante y responde a las necesidades subjetivas de los participantes, por tanto, debe estar basado en dinámicas y procesos contrastados (Gearing et al., 2014; Plan Nacional Sobre Drogas, 2016). Teniendo en cuenta que el compromiso y la implicación en el programa es un factor dinámico que cambia a lo largo del tiempo (Bamberger et al., 2014), se necesitan medir el nivel de participación en las diferentes sesiones y también entre sesiones (Gearing et al., 2014).

Siguiendo con las recomendaciones para futuros estudios, en la línea de lo expresado por el Plan Nacional sobre Drogas (2016) y los resultados de revisiones similares (en Negreiros et al., 2019), se proponen algunas acciones para lograr la adherencia de las familias participantes al programa. Estas recomendaciones van más allá de la adherencia como participación presencial e incluyen propuestas de buenas prácticas relativas al reclutamiento o captación de 
Table 5. Recomendaciones para mejorar la adherencia.

\begin{tabular}{|c|c|}
\hline Reclutamiento & $\begin{array}{l}\text { - Implicación de instituciones reconocidas por las familias y difusión de los programas a través de estas. } \\
\text { - Colaboración con personas reconocidas o líderes de la comunidad. } \\
\text { - Implicación de técnicos y mediadores que ya trabajan con familias. Por ejemplo, la organización de sesiones informativas con } \\
\text { estos técnicos. } \\
\text { - Colaboración de padres de aplicaciones anteriores en sesiones informativas para captación. } \\
\text { - Presentación de los programas resaltando los beneficios de los mismos. } \\
\text { - Procurar que transcurra el tiempo mínimo entre la reunión informativa y la primera sesión. }\end{array}$ \\
\hline Implementación & $\begin{array}{l}\text { - Formación adecuada de los formadores en los contenidos, habilidades de liderazgo y dinamización de grupos. } \\
\text { - Sesiones formativas de refuerzo a los formadores. } \\
\text { - Estabilidad en el equipo formativo a lo largo de la aplicación. } \\
\text { - Creación de ambiente agradable para el desarrollo de las sesiones con las familias. } \\
\text { - Integración de las ideas de los padres. } \\
\text { - Promoción de la escucha activa. } \\
\text { - Fomento de la interacción y la participación para garantizar el aprendizaje de todos. } \\
\text { - Promover el apoyo mutuo entre las familias, destacando las fortalezas del grupo. } \\
\text { - Implementación de sesiones recordatorio para reforzar los aprendizajes. } \\
\text { - Promover y asegurarse de que las familias lleven a cabo en el día a día lo practicado en el programa. } \\
\text { - Responder a las preocupaciones, necesidades y dificultades propias de los participantes. }\end{array}$ \\
\hline
\end{tabular}

Note. Fuente: elaboración propia a partir de PNSD (2016) y Negreiros et al. (2019).

las familias, así como relativas a aspectos de la implementación del programa (véase tabla 5).

Existen muchas razones para intentar mejorar la adherencia a los programas preventivos y la manera en que se informa sobre este constructo. La presente revisión, como principal aportación práctica, proporciona un marco de referencia en cuanto a buenas prácticas y una guía para el establecimiento de un estándar de evaluación de la adherencia en programas de prevención familiar universal.

\section{Reconocimientos}

Este estudio se ha financiado a partir de: EDU201679235-R - "VALIDACION DEL PROGRAMA DE COMPETENCIA FAMILIAR UNIVERSAL 10-14, PCF-U”, 2017-2019. Ministerio de Economía y Competitividad (MINECO).

\section{Conflicto de intereses}

Los autores manifiestan que no hay conflicto de intereses.

\section{Referencias}

Aarons, G. A., Hurlburt, M. y Horwitz, S. M. (2011). Advancing a conceptual model of evidence-based practice implementation in public service sectors. Administration and Policy in Mental Health, 38, 4-23. doi:10.1007/s10488010-0327-7.
Al-Halabi-Diaz, S. y Errasti, P. J. M. (2009). Use of small incentives for increasing participation and reducing dropout in a family drug-use prevention program in a Spanish sample. Substance Use Ẽ Misuse, 44, 1990-2000. doi:10.3109/10826080902844870.

Allen, J. D., Linnan, L. A. y Emmons, K. M. (2012). Fidelity and its relationship to impementation effectiveness, adaptation, and dissem- ination. En R. C. Brownson, G. A. Colditz y E. K. Proctor (Eds.), Dissemination and implementation in health: Translating science to practice (pp. 281304). New York: Oxford University Press.

Axford, N., Lehtonen, M., Tobin, K., Kaoukji, D. y Berry, V. (2012). Engaging parents in parenting programs: Lessons from research and practice. Children and Youth Services R $\boldsymbol{e}_{-}$ view, 34, 2061-2071. doi:10.1016/j.childyouth.2012.06.011.

Baldus, C., Thomsen, M., Sack, P-M., Bröning, S., Arnaud, N., Daubmann, A. y Thomasius, R. (2016). Evaluation of a German version of the Strengthening Families Programme 10-14: A randomised controlled trial. European Journal of Public Health, 26, 953-959. doi:10.1093/eurpub/ckw082.

Ballester, L., Valero, M., Orte, C. y Amer, J. (2018). An analysis of family dynamics: A selective substance abuse prevention programme for adolescents. European Journal of Social Work, 23, 93-105. doi:10.1080/13691457.2018.1473842.

Bamberger, K. T., Coatsworth, J. D., Fosco, G. M. y Ram, N. (2014). Change in participant engagement during a family-based preventive intervention: Ups and downs with 
time and tension. Journal of family psychology: JFP : Journal of the Division of Family Psychology of the American Psychological Association (Division 43), 28, 811-820. doi:10.1037/ fam0000036.

Bodin, M. C. y Strandberg, A. K. (2011). The Örebro prevention programme revisited: A cluster-randomized effectiveness trial of programme effects on youth drinking. Addiction, 106, 2134-2143. doi:10.1111/j.13600443.2011.03540.x.

Bröning, S., Baldus, C., Thomsen, M., Sack, P.M., Arnaud, N. y Thomasius, R. (2017). Children with elevated psychosocial risk load benefit most from a family-based preventive intervention: Exploratory differential analyses from the German "Strengthening Families Program 10-14" adaptation trial. Prevention Science, 18, 932-942. doi:10.1007/s11121-017-0797-x.

Byrnes, H. F., Miller, B. A., Aalborg, A. E., Plasencia, A. V. y Keagy, C. D. (2010). Implementation fidelity in adolescent family-based prevention programs: Relationship to family engagement. Health Education Research, 25, 531541. doi:10.1093/her/cyq006.

Coombes, L., Allen, D. M. y Foxcroft, D. (2012). An exploratory pilot study of the Strengthening Families Programme 10-14 (UK). Drugs: Education, Prevention $\mathcal{E}^{\circ}$ Policy, 19, 387-396. doi:10.3109/09687637.2012.658889.

Errasti, P. J. M., Al-Halabi-Díaz, S., Secades, V. R., Fernández-Hermida, J.R., Carballo, J. L. y García-Rodríguez, O. (2009). Prevención familiar del consumo de drogas: El programa «Familias que funcionan». Psicothema, 21, 45-50.

Fosco, G. M., Frank, J. L., Stormshak, E. A. y Dishion, T. J. (2013). Opening the "Black Box": Family Check-Up intervention effects on self-regulation that prevents growth in problem behavior and substance use. Journal of School Psychology, 51, 455-468. doi:10.1016/j.jsp.2013.02.001.

Foxcroft, D. R., Callen, H., Davies, E. L. y Okulicz-Kozaryn, K. (2017). Effectiveness of the strengthening families programme 10-14 in Poland: Cluster randomized controlled trial. European Journal of Public Health, 27, 494500. doi:10.1093/eurpub/ckw195.

García del Castillo, J.A., García del Castillo-López, A. y López-Sánchez, C. (2014). Concepto de adherencia preventiva en el ámbito de las adicciones. Health and Addictions, 14, 89-98.

Gearing, R. E., Townsend, L., Elkins, J., El-Bassel, N. y Osterberg, L. (2014). Strategies to predict, measure, and improve psychosocial treatment adherence. Harvard Review of Psychiatry, 22, 31-45. doi:10.1097/HRP.10.1097/ HRP.0000000000000005.

Gottfredson, D. C., Cook, T. D., Gardner, F. E. M., Gorman-Smith, D., Howe, G. W., Sandler, I. N. y Zafft, K. M. (2015). Standards of evidence for efficacy, effectiveness, and scale-up research in prevention science: Next generation. Prevention Science, 16, 893-926. doi:10.1007/ s11121-015-0555-x.
Guilamo-Ramos, V., Jaccard, J., Dittus, P., Gonzalez, B., Bouris, A. y Banspach, S. (2010). The Linking Lives Health Education Program: A randomized clinical trial of a parent-based tobacco use prevention program for african american and latino youths. American Journal of Public Health, 100, 1641-1647. doi:10.2105/AJPH.2009.171637.

Guyll, M, Spoth, R. y Cornish, M. (2012). Substance misuse prevention and economic analysis: Challenges and opportunities regarding international utility. Substance Use $\mathcal{E}$ Misuse, 47, 8-9, 877.

Haevelmann, A., Bröning, S., Klein, M., Moesgen, D., Wartberg, L. y Thomasius, R. (2013). Empirical quality assurance in the evaluation of "trampoline" - A group intervention for children of substance-using parents. Suchttherapie, 14, 128-134. doi:10.1055/s-0033-1349098.

Haggerty, K., Skinner, M., MacKenzie, E. y Catalano, R. (2007). A randomized trial of parents who care: Effects on key outcomes at 24-month follow-up. Prevention Science, 8, 249-260. doi:10.1007/s11121-007-0077-2.

Haggerty, K., Skinner, M., Catalano, R., Abbott, R. y Crutchfield, R. (2015). Long-term effects of staying connected with your teen on drug use frequency at age 20. Prevention Science, 16, 538-549. doi:10.1007/s11121-014-0525-8.

Koning, I.M., Volleberg, W.A. M., Smit, F., Verdurmen, J.E.E., Van den Eijden, R.J.J.M., Ter Bogt, T.F.,... Engels, R.C.M.E. (2009). Preventing heavy alcohol use in adolescence (PAS): Cluster randomized trial of a parent and student intervention offered separately and simultaneously. Addiction, 104, 1669-1678. doi:10.1111/j.13600443.2009.02677.x.

Koning, I. M., van den Eijnden, R. J., Verdurmen, J. E., Engels, R. C. y Vollebergh, W. A. (2011). Long-term effects of a parent and student intervention on alcohol use in adolescents: A cluster randomized controlled trial. American Journal of Preventive Medicine, 40, 541-547. doi:10.1016/j.amepre.2010.12.030.

Koning, I. M., van den Eijnden, R. J. J. M., Verdurmen, J. E. E., Engels, R. C. M. E. y Vollebergh, W. A. M. (2013). A cluster randomized trial on the effects of a parent and student intervention on alcohol use in adolescents four years after baseline; No evidence of catching-up behavior. Addictive Behaviors, 38, 2032-2039. doi:10.1016/j. addbeh.2012.12.013.

Koutakis, N., Stattin, H. y Kerr, M. (2008). Reducing youth alcohol drinking through a parent-targeted intervention: The Örebro Prevention Program. Addiction, 103, 1629-1637. doi:10.1111/j.1360-0443.2008.02326.x.

Kumpfer, K.L. (2008). Why are there no effective child abuse prevention parenting interventions? Substance Use $\mathcal{E}$ Misuse, 43, 1262-1265. doi:10.1080/10826080802215114.

Lloret, I. D., Espada, S. J., Cabrera, P. V. y Burkhart, G. (2013). Prevención familiar del consumo de drogas en Europa: Una revisión crítica de los programas contenidos en EDDRA. Adicciones, 25, 226-234. 
Negreiros, J. (2013). Participación parental en intervenciones familiares de toxicodependencias: Una revisión bibliográfica empírica. Pedagogía Social. Revista Interuniversitaria, 21, 39-65. doi:10.7179/psri_2013.21.2.

Negreiros, J., Ballester, L., Valero, M., Carmo, R. y da Gama, J. (2019). Una revisión sistemática de la participación en los programas de prevención familiar. Pedagogía Social. Revista Interuniversitaria, 34, 63-75. doi:10.7179/PSRI_2019.34.05.

Orte, C., Ballester, L., Amer, J. y Vives, M. (2014). Assessing the role of facilitators in evidence-based family-centric prevention programs via delphi technique. Families in Society: The Journal of Contemporary Social Services, 95, 236244. doi:10.1606/1044-3894.2014.95.30.

Plan Nacional Sobre Drogas. (2016). Acción 4: Proyecto coordinado de prevención familiar universal. Guía para la implantación y desarrollo de programas de prevención familiar universal de calidad. Madrid: Plan Nacional Sobre Drogas. Recuperado de http://www.pnsd.mscbs.gob.es/pnsd/ planAccion/plan/productos/pdf/Accion_4_GUIA.pdf.

Rial, A., Burkhart, G., Isorna, M., Barreiro, C., Varela, J. y Golpe, S. (2019). Cannabis use among adolescents: Risk pattern, implications and possible explanatory variables. Adicciones, 31, 64-77. doi:10.20882/adicciones.1212.

Riesch, S. K., Brown, R. L., Anderson, L. S., Wang, K., Canty-Mitchell, J. y Johnson, D. L. (2011). Strengthening Families Program (10-14). Western Journal of Nursing Research, 34, 340-376. doi:10.1177/0193945911399108.

Rosenman, R. E., Goates, S. y Hill, L. (2012). Participation in universal prevention programmes. Applied Economics, 44, 219-228. doi:10.1080/00036846.2010.502111.

Sánchez-Meca, J. y Botella, J. (2015). Meta-análisis en ciencias sociales y de la salud. Madrid: Editorial Síntesis.

Schwalbe, C. y Gearing, R. (2012). The moderating effect of adherence-promoting interventions with clients on evidence-based practices for children and adolescents with mental health problems. American Journal of Orthopsychiatry, 82, 146-155. doi:10.1111/j.19390025.2011.01133.x.

Segrott, J., Murphy, S., Rothwell, H., Scourtfield, J., Foxcroft, D., Gillespie, D.,... Moore, L. (2017). An application of Extended Normalisation Process Theory in a randomised controlled trial of a complex social intervention: Process evaluation of the Strengthening Families Programme (10-14) in Wales, UK. SSM-Population Health, 3, 255-265. doi:10.1016/j.ssmph.2017.01.002.

Sexton, T. y Turner, C.W. (2010). The effectiveness of functional family therapy for youth with behavioral problems in a community practice setting. Journal of Family Psychology, 24, 339-348. doi:10.1037/a0019406.

Skärstrand, E., Sundell, K. y Andréasson, S. (2014). Evaluation of a Swedish version of the Strengthening Families Programme. European Journal of Public Health, 24, 578584. doi:eurpub/ckt146.
Spoth, R. L. y Redmond, C. (2002). Project family prevention trials based in community-university partnerships: Toward scaled-up preventive interventions. Prevention Science, 3, 203-221. doi:10.1023/A:1019946617140.

Spoth, R., Trudeau, L., Shin, C. y Redmond, C. (2008a). Long-term effects of universal preventive interventions on prescription drug misuse. Addiction, 103, 1160-1168. doi:10.1111/j.1360-0443.2008.02160.x.

Spoth, R. L., Randall, G. K., Trudeau, L., Shin, C. y Redmond, C. (2008b). Substance use outcomes $5^{1 / 2}$ years past baseline for partnership-based, family-school preventive interventions. Drug and Alcohol Dependence, 96, 57-68.

Spoth, R., Trudeau, L., Shin, C., Randall, G. K. y Mason, W. A. (2018). Testing a model of universal prevention effects on adolescent relationships and marijuana use as pathways to young adult outcomes. Journal of Youth and Adolescence, 48, 444-458. doi:10.1007/s10964-018-0946-y.

Stormshak, E. A., Connell, A. M., Véronneau, M.-H., Myers, M. W., Dishion, T. J., Kavanagh, K. y Caruthers, A. S. (2011). An ecological approach to promoting early adolescent mental health and social adaptation: Family-centered intervention in public middle schools. Child Development, 82, 209-225. doi:10.1111/j.14678624.2010.01551.x.

Teixidó-Compañó, E., Sordo, L., Bosque-Prous, M., Puigcorbé, S., Barrio, G., Brugal, M.,... Espelt, A. (2019). Factores individuales y contextuales relacionados con el binge drinking en adolescentes españoles: Un enfoque multinivel. Adicciones, 31, 41-51. doi:10.20882/adicciones.975.

Urrútia, G. y Bonfill, X. (2010). Declaración PRISMA: Una propuesta para mejorar la publicación de revisions sistemáticas y meta-análisis. Medicina Clínica, 135, 507-511. doi:10.1016/j.medcli.2010.01.015

Van Ryzin, M. J., Stormshak, E. A. y Dishion, T. J. (2012). Engaging parents in the Family Check-Up in middle school: Longitudinal effects on family conflict and problem behavior through the high school transition. Journal of Adolescent Health, 50, 627-633. doi:10.1016/j. jadohealth.2011.10.255.

Verdurmen, J. E. E., Koning, I. M., Vollebergh, W. A. M., van den Eijnden, R. J. J. M. y Engels, R. C. M. E. (2014). Risk moderation of a parent and student preventive alcohol intervention by adolescent and family factors: A cluster randomized trial. Preventive Medicine: An International Journal Devoted to Practice and Theory, 60, 88-94. doi:10.1016/j.ypmed.2013.12.02. 